\title{
Seletividade de herbicidas aplicados em pré-emergência na cultura do algodão'
}

\author{
Selectivity of herbicides applied to pre-emergent cotton crops
}

\author{
Miriam Hiroko Inoue ${ }^{2 *}$, Rubém Silvério de Oliveira Junior ${ }^{3}$, Ronei Ben ${ }^{2}$, Rivanildo Dallacort $^{2}$ e Cassiano Luiz \\ Sztoltz ${ }^{2}$
}

\begin{abstract}
RESUMO - A cultura do algodão tem grande importância econômica e social, visto que é a fibra mais utilizada no setor têxtil. Contudo é uma cultura que apresenta alta sensibilidade a plantas daninhas e há poucos herbicidas seletivos à cultura. Neste contexto o trabalho objetivou avaliar a seletividade de herbicidas aplicados em pré-emergência na cultura do algodão. Os herbicidas alachlor, S-metolachlor, diuron, prometryne, trifluralin e oxyfluorfen foram aplicados isoladamente e em misturas sobre a variedade FMT-701, nas localidades de Diamantino-MT e Campos de Júlio-MT. O delineamento experimental utilizado foi em blocos casualizados com 16 tratamentos e 4 repetições. Para avaliar a seletividade foram realizadas avaliações de altura aos 36; 66 e 150 dias após a aplicação (DAA), fitointoxicação aos 14; $21 ; 29$ e 36 DAA, estande aos 21 e 49 DAA, número de maçãs aos 141 DAA e produtividade do algodão em caroço aos 193 DAA. Os dados foram submetidos à análise conjunta e ao teste de agrupamento Scott-Knott $(\mathrm{p}>0,05)$. Os resultados indicaram que alguns tratamentos proporcionaram menor altura de plantas em determinadas avaliações e grande parte dos tratamentos causou injúrias na fase inicial da cultura. Verificou-se que os tratamentos não proporcionaram diferença significativa para as características de estande de plantas, número de maçãs e produtividade de algodão em caroço, comprovando que todos os tratamentos avaliados podem ser utilizados no manejo de plantas daninhas.
\end{abstract}

Palavras-chave: Algodão-cultivo. Ervas daninhas-controle.

\begin{abstract}
The cultivation of cotton has great economic and social importance as it is the most widely used fibre in the textile sector. It is however a crop that is highly sensitive tweeds, and there are few selective herbicides for the crop. With this in mind, this study aimed to evaluate the selectivity of herbicides applied to pre-emergent cotton crops. The herbicides, alachlor, S-metolachlor, diuron, prometryne, trifluralin and oxyfluorfen were applied both individually and in mixture to the variety FMT-701 in the towns of Diamantino and Campos de Júlio, Mato Grosso. The experimental design used was of randomized blocks with 16 treatments and four replications. To evaluate selectivity, assessments were made for height at 36, 66 and 150 days after application (DAA), for phytotoxicity at 14, 21, 29 and 36 DAA, for growth at 21 and 49 DAA, for the number of bolls at $141 \mathrm{DAA}$ and for cotton-seed yield at $193 \mathrm{DAA}$. The data were submitted to joint-analysis and the ScottKnott cluster test $(p>0.05)$. The results indicated that some treatments resulted in lower plant height in certain evaluations, and that a large part of the treatments caused injury in the initial stage of cultivation. It was found that the treatments did not produce significant differences for the characteristics of plant growth, number of bolls and cotton-seed yield, proving that all the treatments assessed may be used in weed management.
\end{abstract}

Key words: Cotton-cultivation. Weed-control.

\footnotetext{
* Autor para correspondência

${ }^{1}$ Recebido para publicação em 15/09/2011; aprovado em 19/06/2012

Parte da Monografia do quinto autor, apresentada ao Departamento de Agronomia da Universidade do Estado de Mato Grosso - UNEMAT

${ }^{2}$ Departamento de Agronomia, Universidade do Estado de Mato Grosso/UNEMAT, Tangará da Serra-MT, Brasil, miriamhinoue@hotmail.com, roneiben@hotmail.com, rivanildo@unemat.br, cassianocls@gmail.com

${ }^{3}$ Departamento de Agronomia, Universidade Estadual de Maringá/UEM, Maringá-PR, Brasil, rsojunior@uem.br
} 


\section{INTRODUÇÃO}

A área plantada com algodão no País para a safra 2010/11 chega à marca dos 1.400,3 mil hectares, superior em $67,6 \%$ à cultivada na safra 2009/10. Em valores absolutos representam 564,6 mil hectares a mais. A alta no preço e a forte redução dos estoques mundiais são fatores que justificam tal incremento. O maior incremento de área ocorre na região Centro-Oeste, que participa com $63,8 \%$ no total da área plantada. Em Mato Grosso, principal estado produtor, o crescimento na área ocorreu principalmente no plantio de primeira safra, provocado, sobretudo, pelo retardamento do plantio da soja (COMPANHIA DE ABASTECIMENTO, 2011).

Segundo Ballaminut (2009), o algodoeiro é uma espécie bastante sensível à interferência imposta por plantas daninhas, destacando-se a competição por fatores de crescimento (água, luz e nutrientes), a liberação de substâncias alelopáticas e a multiplicação de insetospraga e doenças, que retardam o desenvolvimento da cultura. Além disso, as plantas daninhas podem promover decréscimo na qualidade do produto colhido e dificultar a realização da colheita da cultura (CARDOSO et al., 2010; FREITAS, 2003).

Nos cerrados, as áreas agrícolas são normalmente constituídas de grandes áreas de plantio, nas quais são utilizadas práticas de manejo de plantas daninhas basicamentepormeiodocontrolequímico(GUIMARÃES; HRYCYK; MENDONÇA, 2007). Apesar de eficientes, há poucas opções de herbicidas seletivos aplicados em pré-emergência para o algodoeiro, sendo realizadas aplicações com produtos que resultam, muitas vezes, em injúrias à cultura e baixa qualidade de fibra e rendimento do algodoeiro (FOLONI; RODRIGUES; ONO, 1999; FREITAS et al., 2006a).

Atualmente o cultivo do algodoeiro se concentra nos estados de Mato Grosso e no Oeste da Bahia (INOUE et al., 2010). Particularmente no Mato Grosso, muitas áreas de cultivo apresentam problemas com plantas daninhas de difícil controle, o que aumenta a necessidade de estudar o efeito das doses e a seletividade de herbicidas nessas áreas (FREITAS et al., 2006b).

Dentro desse contexto, são fundamentais os estudos que visem avaliar a seletividade de herbicidas. Embora muitas vezes os estudos relacionados à seletividade se restrinjam à avaliação de sintomas visuais, existem produtos que afetam a produtividade da cultura sem demonstrar sintomas visuais e outros que, por sua vez, provocam injúrias acentuadas, mas que permitem a cultura manifestar plenamente seu potencial produtivo (SOCIEDADE BRASILEIRA DA CIÊNCIA DAS PLANTAS DANINHAS, 1995).
Diante dos fatos mencionados, este trabalho teve como objetivo avaliar a seletividade de herbicidas, aplicados em pré-emergência, isolados e em misturas, na cultura do algodão.

\section{MATERIAL E MÉTODOS}

Dois experimentos com os mesmos tratamentos foram conduzidos simultaneamente em duas áreas distintas: a primeira localizada na Fazenda Campo Alegre, Rodovia MT 364, km 330, município de Diamantino-MT (144'54" S, 57 $34^{\circ} 41^{\prime \prime}$ W e altitude de $650 \mathrm{~m}$ ), e a segunda na Fazenda Juína, no município de Campos de Júlio-MT (13²6' '" S, 59²15'18" W e altitude de 225 m). A região do município de Diamantino apresenta uma precipitação anual de $1.750 \mathrm{~mm}$ com temperatura média anual de $24^{\circ} \mathrm{C}$, e a região do município de Campos de Júlio a precipitação anual fica em torno dos $2.500 \mathrm{~mm}$ com uma temperatura média anual de $24{ }^{\circ} \mathrm{C}$ (FERREIRA; SILVA, 2008). Os solos de cada área foram classificados, respectivamente, como Latossolo Vermelho (textura argilosa) e Nitossolo Vermelho (textura muito argilosa) (EMPRESA BRASILEIRA DE PESQUISA AGROPECUÁRIA, 2006), e suas principais características químicas e físicas estão descritas na Tabela 1 .

Em ambos os experimentos, a adubação de base foi realizada no sulco de semeadura de acordo com as análises de solo. Imediatamente antes da semeadura, as sementes foram tratadas com o safener Permit, na dose de $1,2 \mathrm{~kg} 100 \mathrm{~kg}^{-1}$ de sementes.

A semeadura da cultura do algodão foi realizada em 09/12/2008, utilizando semeadora com seis linhas e densidade de 10 a 12 sementes por metro linear e profundidade aproximada de $2,0 \mathrm{~cm}$. Cada unidade experimental foi constituída por cinco linhas de semeadura, 5,0 $\mathrm{m}$ de comprimento e espaçamento entre linhas de $0,9 \mathrm{~m}$, totalizando $22,5 \mathrm{~m}^{2}$. Para as avaliações foram descartadas as bordaduras, utilizando como área útil as três linhas centrais, desconsiderando-se o metro inicial e final de cada parcela $\left(8,1 \mathrm{~m}^{2}\right)$. A variedade semeada foi a FMT-701.

Foram avaliados 15 tratamentos com os herbicidas alachlor, S-metolachlor, diuron, prometryne, trifluralin e oxyfluorfen, aplicados isoladamente e em misturas, além da testemunha sem aplicação de herbicida. A aplicação dos herbicidas foi realizada um dia após a semeadura, por meio de pulverizador costal de pressão constante à base de $\mathrm{CO}_{2}$, sob pressão de $2 \mathrm{kgf} \mathrm{cm}^{-2}$, equipado com cinco pontas XR 110.02, espaçadas de $0,5 \mathrm{~m}$, proporcionando volume de calda equivalente a $200 \mathrm{~L} \mathrm{ha}^{-1}$. 
Tabela 1 - Características químicas e físicas dos solos presentes nas áreas em que os experimentos foram conduzidos

\begin{tabular}{|c|c|c|c|c|c|c|c|}
\hline \multirow{2}{*}{ Solo } & \multicolumn{2}{|c|}{$\mathrm{pH}$} & $\mathrm{Al}^{3+}$ & $\mathrm{H}^{+}+\mathrm{Al}^{3+}$ & $\mathrm{Ca}^{+2}+\mathrm{Mg}^{2+}$ & $\mathrm{Ca}^{2+}$ & $\mathrm{K}^{+}$ \\
\hline & $\mathrm{CaCl}_{2}$ & $\mathrm{H}_{2} \mathrm{O}$ & ----------- & 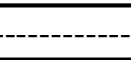 & $-\mathrm{cmol}_{\mathrm{c}} \mathrm{dm}^{-3}$ & 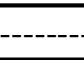 & 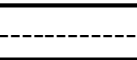 \\
\hline Área ${ }^{1 /}$ & 5,20 & 5,90 & 0,00 & 3,90 & 3,60 & 2,80 & 0,13 \\
\hline Área ${ }^{2 /}$ & 5,20 & 6,00 & 0,00 & 4,60 & 4,75 & 2,81 & 0,12 \\
\hline \multirow{2}{*}{ Solo } & $\mathrm{P}$ & M.O. & CTC & $\mathrm{V}$ & areia & silte & Argila \\
\hline & $\mathrm{mg} \mathrm{dm} \mathrm{m}^{-3}$ & $\mathrm{~g} \mathrm{dm}^{-3}$ & $\mathrm{cmol}_{\mathrm{c}} \mathrm{dm}^{-3}$ & $\%$ & \multicolumn{3}{|c|}{ - - } \\
\hline Área ${ }^{1 /}$ & 10,20 & 27,70 & 7,60 & 48,90 & 249 & 150 & 601 \\
\hline Área ${ }^{2 \prime}$ & 7,10 & 30,00 & 9,50 & 51,50 & 168 & 92 & 740 \\
\hline
\end{tabular}

1/Amostra proveniente de Diamantino, Latossolo Vermelho distroférrico (textura argilosa); ’'Amostra proveniente de Campos de Júlio, Nitossolo Vermelho (textura muito argilosa)

Os tratamentos foram: alachlor $\left(1,68 \mathrm{~kg} \mathrm{ha}^{-1}\right)$, S-metolachlor $\left(0,96 \mathrm{~kg} \mathrm{ha}^{-1}\right)$, diuron $\left(1,75 \mathrm{~kg} \mathrm{ha}^{-1}\right)$, prometryne $\left(1,75 \mathrm{~kg} \mathrm{ha}^{-1}\right)$, trifluralin $\left(2,10 \mathrm{~kg} \mathrm{ha}^{-1}\right)$, oxyfluorfen $\left(0,24 \mathrm{~kg} \mathrm{ha}^{-1}\right)$, alachlor + diuron $(1,68+1,75$ $\left.\mathrm{kg} \mathrm{ha}^{-1}\right)$, alachlor + prometryne $\left(1,68+1,75 \mathrm{~kg} \mathrm{ha}^{-1}\right), \mathrm{S}-$ metolachlor + diuron $\left(0,96+1,75 \mathrm{~kg} \mathrm{ha}^{-1}\right), \mathrm{S}$-metolachlor + prometryne $\left(0,96+1,75 \mathrm{~kg} \mathrm{ha}^{-1}\right)$, oxyfluorfen + diuron $\left(0,24+1,75 \mathrm{~kg} \mathrm{ha}^{-1}\right)$, oxyfluorfen + prometryne $(0,24+$ $\left.1,75 \mathrm{~kg} \mathrm{ha}^{-1}\right)$, trifluralin + diuron $\left(2,10+1,75 \mathrm{~kg} \mathrm{ha}^{-1}\right)$, trifluralin + prometryne $\left(2,10+1,75 \mathrm{~kg} \mathrm{ha}^{-1}\right)$, trifluralin + oxyfluorfen $\left(2,10+0,24 \mathrm{~kg} \mathrm{ha}^{-1}\right)$ e testemunha sem tratamento com herbicida.

Independente do tratamento avaliado, todas as parcelas foram mantidas isentas da presença de plantas daninhas durante o ciclo da cultura, por meio de capinas manuais. Os tratos culturais referentes a pragas, doenças, adubações de cobertura foram realizadas de acordo com os monitoramentos e recomendações agronômicas. A fim de evitar o crescimento excessivo do algodoeiro, foram feitas três aplicações de $15 \mathrm{~g} \mathrm{ha}^{-1}$ do regulador de crescimento cloreto de mepiquat, sendo a primeira aos 45 dias após a emergência e as demais em intervalos de 15 dias.

Durante o desenvolvimento da cultura, foram realizadas avaliações de altura, fitointoxicação, estande, número de maçãs e produtividade do algodão em caroço. As avaliações de altura foram feitas aos 36; 66 e 150 DAA, utilizando-se aleatoriamente dez plantas por parcela e medindo desde o colo até a inserção da folha mais nova completamente expandida na planta (GOULART, 2002). As avaliações de fitointoxicação da cultura foram realizadas aos $14 ; 21 ; 29$ e 36 dias após a aplicação (DAA), atribuindo-se notas de 1 a 9, correspondendo a nenhum sintoma e morte total, respectivamente (SOCIEDADE BRASILEIRA DA CIÊNCIA DAS PLANTAS DANINHAS, 1995).
A avaliação de estande foi realizada aos 21 e 49 DAA, contando-se na segunda linha de plantio o número de plantas presentes em 5,0 m de cada parcela (CRUSCIOL et al., 2002). Na avaliação de maçãs, foram contadas o número de maçãs presentes em cinco plantas selecionadas aleatoriamente na parcela aos 141 DAA. Para a produtividade do algodão em caroço foi realizada a colheita da área útil das parcelas aos 193 DAA.

Em ambos os experimentos, foi utilizado o delineamento experimental em blocos casualizados com quatro repetições. Os dados obtidos nas avaliações foram submetidos à análise de variância conjunta e, na presença de interação significativa, procederam-se os desdobramentos necessários ( $\mathrm{p}>0,05)$. As médias foram comparadas pelo teste de agrupamento de Scott e Knott (1974). Foi realizada análise conjunta dos dados, uma vez que a razão entre o maior e o menor quadrado médio residual não foi superior a sete (BANZATTO; KRONKA, 1995).

\section{RESULTADOS E DISCUSSÃO}

A análise conjunta dos dados revelou que a interação tratamentos $x$ locais foi significativa $(p>0,05)$ para as variáveis altura de plantas (36 e 66 DAA), fitointoxicação (14; 21 e 29 DAA), número de maçãs e produtividade. A Tabela 2 apresenta os resultados do desdobramento para avaliação de altura de plantas aos 36 DAA. Observa-se que em Diamantino não houve efeito na altura das plantas em comparação com a testemunha sem herbicida, nos tratamentos com diuron, prometryne, trifluralin + diuron e trifluralin + prometryne. As plantas nos demais tratamentos apresentaram redução entre 6 e $36 \%$ na sua altura, em relação à testemunha. 
Tabela 2 - Avaliação de altura (cm) aos 36 dias após aplicação (DAA) dos tratamentos com herbicidas em pré-emergência. Diamantino e Campos de Júlio - MT. Safra 2008/2009

\begin{tabular}{lcccc}
\hline \multirow{2}{*}{ Tratamentos (i.a.) } & kg i.a. ha ${ }^{-1}$ & \multicolumn{2}{c}{ Altura das plantas (cm) } & Médias \\
\cline { 3 - 4 } & & Diamantino & C. de Júlio & \\
\hline 1. alachlor & 1,68 & $20,60 \mathrm{Cb}$ & $30,72 \mathrm{Ba}$ & 25,66 \\
2. S-metolachlor & 0,96 & $18,22 \mathrm{Cb}$ & $31,12 \mathrm{Ba}$ & 24,67 \\
3. diuron & 1,75 & $24,67 \mathrm{Ab}$ & $31,57 \mathrm{Ba}$ & 28,12 \\
4. prometryne & 1,75 & $25,27 \mathrm{Ab}$ & $33,37 \mathrm{Aa}$ & 29,32 \\
5. trifluralin & 2,10 & $23,37 \mathrm{Bb}$ & $31,42 \mathrm{Ba}$ & 27,40 \\
6. oxyfluorfen & 0,24 & $22,17 \mathrm{Bb}$ & $28,05 \mathrm{Ba}$ & 25,11 \\
7. alachlor + diuron & $1,68+1,75$ & $15,82 \mathrm{Db}$ & $32,20 \mathrm{Aa}$ & 24,01 \\
8. alachlor + prometryne & $1,68+1,75$ & $16,95 \mathrm{Db}$ & $30,80 \mathrm{Ba}$ & 23,88 \\
9. S-metolachlor + diuron & $0,96+1,75$ & $18,10 \mathrm{Cb}$ & $31,00 \mathrm{Ba}$ & 24,55 \\
10. S-metolachlor + prometryne & $0,96+1,75$ & $19,00 \mathrm{Cb}$ & $32,65 \mathrm{Aa}$ & 25,83 \\
11. oxyfluorfen + diuron & $0,24+1,75$ & $22,55 \mathrm{Bb}$ & $30,12 \mathrm{Ba}$ & 26,34 \\
12. oxyfluorfen + prometryne & $0,24+1,75$ & $22,07 \mathrm{Bb}$ & $29,47 \mathrm{Ba}$ & 25,77 \\
13. trifluralin + diuron & $2,10+1,75$ & $24,72 \mathrm{Ab}$ & $34,65 \mathrm{Aa}$ & 29,69 \\
14. trifluralin + prometryne & $2,10+1,75$ & $24,17 \mathrm{Ab}$ & $34,02 \mathrm{Aa}$ & 29,10 \\
15. trifluralin + oxyfluorfen & $2,10+0,24$ & $21,65 \mathrm{Bb}$ & $29,45 \mathrm{Ba}$ & 25,55 \\
16. testemunha sem herbicida & - & $24,86 \mathrm{Ab}$ & $33,25 \mathrm{Aa}$ & 29,06 \\
\hline \multicolumn{1}{c}{ Médias } & \multicolumn{5}{c}{21,51} & 31,49 & \\
\hline \multicolumn{1}{c}{ C.V. (\%) } & 6,59 & & \\
\hline Médias seguidas de mesma letra maiúscula na coluna e minúscula na linha não diferem entre si pelo teste de agrupamento Scott-Knott (1974) a 5\%
\end{tabular}

Em Campos de Júlio não houve redução na altura das plantas nos tratamentos com prometryne, alachlor + diuron, S-metolachlor + prometryne, trifluralin + diuron e trifluralin + prometryne em relação à testemunha sem herbicida (Tabela 2). Nos demais tratamentos, as plantas tiveram redução de até $16 \%$ na altura. Em relação ao alachlor, resultados semelhantes foram obtidos em casa-de-vegetação por Guimarães, Hrycyk e Mendonça. (2007), utilizando solo com $634 \mathrm{~g} \mathrm{~kg}^{-1}$ de argila, após a aplicação de dose de $2,88 \mathrm{~kg} \mathrm{ha}^{-1}$.

Na Tabela 3 estão dispostos os resultados do desdobramento para avaliação de altura de plantas aos 66 DAA. Para Diamantino, os resultados são semelhantes aos obtidos na avaliação realizada aos 36 DAA. Maior redução (27\%) foi observada quando da utilização de alachlor + prometryne, em relação à testemunha. Em Campos de Júlio, todos os tratamentos foram considerados seletivos para essa variável, não diferindo da testemunha (Tabela 3). Ballaminut (2009) ao avaliarem diuron $\left(2 \mathrm{~kg} \mathrm{ha}^{-1}\right)$ em Nitossolo Eutrófico, textura argilosa, não verificaram efeitos negativos na altura de três cultivares de algodão (Fibermax 966, Delta Opal e Aroeira).

Para a altura de plantas aos 150 DAA (Tabela 4), a análise de variância conjunta dos dados revelou efeito não significativo $(\mathrm{p}>0,05)$ para a interação tratamentos $\mathrm{x}$ locais. Porém, a altura média das plantas provenientes de Campos de Júlio foi superior a de Diamantino, o que pode ter ocorrido em função do estande mais elevado na área de Campos de Júlio. Tal fato pode ter proporcionado maior grau de competição por luz e, consequentemente, maior desenvolvimento vertical das plantas (SILVA et al., 2005). Essa constatação também pode ser atribuída às variações edafoclimáticas que ocorreram entre as duas áreas, proporcionando, eventualmente, crescimento diferencial das plantas. 
Tabela 3 - Avaliação de altura (cm) aos 66 dias após aplicação (DAA) dos tratamentos com herbicidas em pré-emergência. Diamantino e Campos de Júlio - MT. Safra 2008/2009

\begin{tabular}{|c|c|c|c|c|}
\hline \multirow{2}{*}{ Tratamentos (i.a.) } & \multirow{2}{*}{ kg i.a. ha ${ }^{-1}$} & \multicolumn{2}{|c|}{ Altura das plantas $(\mathrm{cm})$} & \multirow{2}{*}{ Médias } \\
\hline & & Diamantino & C. de Júlio & \\
\hline 1. alachlor & 1,68 & $66,12 \mathrm{Bb}$ & $79,05 \mathrm{Aa}$ & 72,59 \\
\hline 2. S-metolachlor & 0,96 & $66,65 \mathrm{Bb}$ & $78,40 \mathrm{Aa}$ & 72,53 \\
\hline 3. diuron & 1,75 & $79,92 \mathrm{Aa}$ & $80,77 \mathrm{Aa}$ & 80,35 \\
\hline 4. prometryne & 1,75 & $74,70 \mathrm{Aa}$ & $80,77 \mathrm{Aa}$ & 77,74 \\
\hline 5. trifluralin & 2,10 & $69,72 \mathrm{Bb}$ & $80,45 \mathrm{Aa}$ & 75,09 \\
\hline 6. oxyfluorfen & 0,24 & $70,00 \mathrm{Bb}$ & $84,55 \mathrm{Aa}$ & 77,28 \\
\hline 7. alachlor + diuron & $1,68+1,75$ & $55,52 \mathrm{Cb}$ & $79,67 \mathrm{Aa}$ & 67,6 \\
\hline 8. alachlor + prometryne & $1,68+1,75$ & $55,20 \mathrm{Cb}$ & $78,75 \mathrm{Aa}$ & 66,98 \\
\hline 9. S-metolachlor + diuron & $0,96+1,75$ & $61,07 \mathrm{Cb}$ & $79,14 \mathrm{Aa}$ & 70,11 \\
\hline 10. S-metolachlor + prometryne & $0,96+1,75$ & $57,87 \mathrm{Cb}$ & $84,50 \mathrm{Aa}$ & 71,19 \\
\hline 11. oxyfluorfen + diuron & $0,24+1,75$ & $68,65 \mathrm{Bb}$ & $83,27 \mathrm{Aa}$ & 75,96 \\
\hline 12. oxyfluorfen + prometryne & $0,24+1,75$ & $71,17 \mathrm{Bb}$ & $84,70 \mathrm{Aa}$ & 77,94 \\
\hline 13. trifluralin + diuron & $2,10+1,75$ & $72,85 \mathrm{Ab}$ & $82,40 \mathrm{Aa}$ & 77,63 \\
\hline 14. trifluralin + prometryne & $2,10+1,75$ & $76,17 \mathrm{Ab}$ & $83,60 \mathrm{Aa}$ & 79,89 \\
\hline 15. trifluralin + oxyfluorfen & $2,10+0,24$ & $71,65 \mathrm{Bb}$ & $81,95 \mathrm{Aa}$ & 76,80 \\
\hline 16. testemunha sem herbicida & - & $75,40 \mathrm{Ab}$ & $83,17 \mathrm{Aa}$ & 79,29 \\
\hline Médias & & 68,29 & 81,57 & \\
\hline C.V. $(\%)$ & 5,92 & & & \\
\hline
\end{tabular}

Médias seguidas de mesma letra maiúscula na coluna e minúscula na linha não diferem entre si pelo teste de agrupamento Scott-Knott (1974) a 5\%

Tabela 4 - Avaliação de altura (cm) aos 150 dias após aplicação (DAA) dos tratamentos com herbicidas em pré-emergência. Diamantino e Campos de Júlio - MT. Safra 2008/2009

\begin{tabular}{|c|c|c|c|c|}
\hline \multirow{2}{*}{ Tratamentos (i.a.) } & \multirow{2}{*}{ kg i.a. ha ${ }^{-1}$} & \multicolumn{2}{|c|}{ Altura das plantas $(\mathrm{cm})$} & \multirow{2}{*}{ Médias } \\
\hline & & Diamantino & C. de Júlio & \\
\hline 1. alachlor & 1,68 & 133,23 & 128,25 & $130,74 \mathrm{~A}$ \\
\hline 2. S-metolachlor & 0,96 & 128,58 & 128,00 & $128,29 \mathrm{~A}$ \\
\hline 3. diuron & 1,75 & 127,23 & 122,50 & $124,86 \mathrm{~A}$ \\
\hline 4. prometryne & 1,75 & 115,33 & 129,50 & $122,41 \mathrm{~A}$ \\
\hline 5. trifluralin & 2,10 & 115,65 & 127,50 & $121,57 \mathrm{~A}$ \\
\hline 6. oxyfluorfen & 0,24 & 117,70 & 137,25 & $127,47 \mathrm{~A}$ \\
\hline 7. alachlor + diuron & $1,68+1,75$ & 114,58 & 131,00 & $122,79 \mathrm{~A}$ \\
\hline 8. alachlor + prometryne & $1,68+1,75$ & 120,10 & 130,25 & $125,17 \mathrm{~A}$ \\
\hline 9. S-metolachlor + diuron & $0,96+1,75$ & 127,73 & 132,00 & $129,86 \mathrm{~A}$ \\
\hline 10. S-metolachlor + prometryne & $0,96+1,75$ & 125,68 & 129,50 & $127,59 \mathrm{~A}$ \\
\hline 11. oxyfluorfen + diuron & $0,24+1,75$ & 125,18 & 129,50 & $127,34 \mathrm{~A}$ \\
\hline 12. oxyfluorfen + prometryne & $0,24+1,75$ & 127,93 & 129,00 & $128,46 \mathrm{~A}$ \\
\hline 13. trifluralin + diuron & $2,10+1,75$ & 128,63 & 127,50 & $128,06 \mathrm{~A}$ \\
\hline 14. trifluralin + prometryne & $2,10+1,75$ & 124,83 & 123,00 & $123,91 \mathrm{~A}$ \\
\hline 15. trifluralin + oxyfluorfen & $2,10+0,24$ & 127,18 & 119,00 & $123,09 \mathrm{~A}$ \\
\hline 16. testemunha sem herbicida & - & 122,70 & 127,20 & $124,95 \mathrm{~A}$ \\
\hline Médias & & $123,89 \mathrm{~b}$ & $128,18 \mathrm{a}$ & \\
\hline C.V. $(\%)$ & 6,88 & & & \\
\hline
\end{tabular}

Médias seguidas de mesma letra maiúscula na coluna e minúscula na linha não diferem entre si pelo teste de agrupamento Scott-Knott (1974), a 5\% 
Na Tabela 5 estão dispostas as notas das avaliações de fitointoxicação dos herbicidas aos 14 DAA. Em Diamantino, os tratamentos alachlor, oxyfluorfen, alachlor + diuron, alachlor + prometryne, S-metolachlor + diuron, S-metolachlor + prometryne, oxyfluorfen + diuron, oxyfluorfen + prometryne e trifluralin + oxyfluorfen proporcionaram injúrias acima de 4 na escala EWRC. Nessas plantas, os principais sintomas foram necrose nas margens das folhas acompanhada de deformação nas folhas e brotos. Os demais tratamentos proporcionaram injúrias mais leves.

Guimarães, Hrycyk e Mendonça (2007) não observaram sintomas de fitointoxicação no algodoeiro utilizando solo de cerrado $\left(634 \mathrm{~g} \mathrm{~kg}^{-1}\right.$ de argila) ao avaliarem alachlor $\left(2,88 \mathrm{~kg} \mathrm{ha}^{-1}\right)$. Por outro lado, Azevedo et al. (1993) observaram sintomas de fitointoxicação com a mistura alachlor + diuron $(1,2+$ $1,0 \mathrm{~kg} \mathrm{ha}^{-1}$ ) no algodoeiro cultivado em solo com $2 \%$ de argila. Em Campos de Júlio somente os tratamentos com oxyfluorfen, oxyfluorfen + diuron, oxyfluorfen + prometryne e trifluralin + oxyfluorfen causaram efeito fitotóxico. Os demais tratamentos não proporcionaram injúrias significativas, indicando serem aparentemente seletivos às plantas na presente avaliação.

$\mathrm{Na}$ Tabela 6 encontra-se a avaliação de fitointoxicação aos 21 DAA onde esta não apresenta tratamentos totalmente seletivos à cultura, porém alguns tiveram grande redução no seu efeito fitotóxico. Os tratamentos que tiveram danos fitotóxicos mais severos em Diamantino foram alachlor + diuron, alachlor + prometryne, S-metolachlor + diuron e Smetolachlor + prometryne, os demais tratamentos aparecem com pequenas alterações. Em Campos de Júlio, os resultados foram semelhantes aos constados na Tabela 5 (14 DAA), ou seja, somente os tratamentos com oxyfluorfen, oxyfluorfen + diuron, oxyfluorfen + prometryne e trifluralin + oxyfluorfen que proporcionaram danos significativos nas plantas.

Tabela 5 - Avaliação de fitointoxicação aos 14 dias após aplicação (DAA) dos tratamentos com herbicidas em pré-emergência. Diamantino e Campos de Júlio - MT. Safra 2008/2009

\begin{tabular}{|c|c|c|c|c|}
\hline \multirow{2}{*}{ Tratamentos (i.a.) } & \multirow{2}{*}{ kg i.a. ha ${ }^{-1}$} & \multicolumn{2}{|c|}{ Fitointoxicação } & \multirow{2}{*}{ Médias } \\
\hline & & Diamantino & C. de Júlio & \\
\hline 1. alachlor & 1,68 & $4,25 \mathrm{Aa}$ & $1,00 \mathrm{Bb}$ & 2,63 \\
\hline 2. S-metolachlor & 0,96 & $3,50 \mathrm{Ba}$ & $1,25 \mathrm{Bb}$ & 2,38 \\
\hline 3. diuron & 1,75 & $2,50 \mathrm{Ba}$ & $1,75 \mathrm{Ba}$ & 2,13 \\
\hline 4. prometryne & 1,75 & $2,50 \mathrm{Ba}$ & $1,50 \mathrm{Ba}$ & 2,00 \\
\hline 5. trifluralin & 2,10 & $2,00 \mathrm{Ca}$ & $1,25 \mathrm{Ba}$ & 1,63 \\
\hline 6. oxyfluorfen & 0,24 & $5,75 \mathrm{Aa}$ & $3,50 \mathrm{Ab}$ & 4,63 \\
\hline 7. alachlor + diuron & $1,68+1,75$ & $5,25 \mathrm{Aa}$ & $2,00 \mathrm{Bb}$ & 3,63 \\
\hline 8. alachlor + prometryne & $1,68+1,75$ & $5,00 \mathrm{Aa}$ & $1,75 \mathrm{Bb}$ & 3,38 \\
\hline 9. S-metolachlor + diuron & $0,96+1,75$ & $5,75 \mathrm{Aa}$ & $2,00 \mathrm{Bb}$ & 3,88 \\
\hline 10. S-metolachlor + prometryne & $0,96+1,75$ & $4,75 \mathrm{Aa}$ & $1,50 \mathrm{Bb}$ & 3,13 \\
\hline 11. oxyfluorfen + diuron & $0,24+1,75$ & $5,50 \mathrm{Aa}$ & $3,25 \mathrm{Ab}$ & 4,38 \\
\hline 12. oxyfluorfen + prometryne & $0,24+1,75$ & $4,25 \mathrm{Aa}$ & $3,00 \mathrm{Ab}$ & 3,63 \\
\hline 13. trifluralin + diuron & $2,10+1,75$ & $3,50 \mathrm{Ba}$ & $1,75 \mathrm{Bb}$ & 2,63 \\
\hline 14. trifluralin + prometryne & $2,10+1,75$ & $1,75 \mathrm{Ca}$ & $1,25 \mathrm{Ba}$ & 1,50 \\
\hline 15. trifluralin + oxyfluorfen & $2,10+0,24$ & $4,75 \mathrm{Aa}$ & $2,50 \mathrm{Ab}$ & 3,63 \\
\hline 16. testemunha sem herbicida & - & $1,00 \mathrm{Ca}$ & $1,00 \mathrm{Ba}$ & 1,00 \\
\hline Médias & & 3,88 & 1,89 & \\
\hline C.V. $(\%)$ & 29,15 & & & \\
\hline
\end{tabular}

Médias seguidas de mesma letra maiúscula na coluna e minúscula na linha não diferem entre si pelo teste de agrupamento Scott-Knott (1974) a 5\% 
Tabela 6 - Avaliação de fitointoxicação aos 21 dias após aplicação (DAA) dos tratamentos com herbicidas em pré-emergência. Diamantino e Campos de Júlio - MT. Safra 2008/2009

\begin{tabular}{|c|c|c|c|c|}
\hline \multirow{2}{*}{ Tratamentos (i.a.) } & \multirow{2}{*}{ kg i.a. ha ${ }^{-1}$} & \multicolumn{2}{|c|}{ Fitointoxicação } & \multirow{2}{*}{ Médias } \\
\hline & & Diamantino & C. de Júlio & \\
\hline 1. alachlor & 1,68 & $2,75 \mathrm{Ca}$ & $1,50 \mathrm{Bb}$ & 2,13 \\
\hline 2. S-metolachlor & 0,96 & $3,50 \mathrm{Ba}$ & $1,75 \mathrm{Bb}$ & 2,63 \\
\hline 3. diuron & 1,75 & $1,75 \mathrm{Da}$ & $1,75 \mathrm{Ba}$ & 1,75 \\
\hline 4. prometryne & 1,75 & $1,25 \mathrm{Da}$ & $2,00 \mathrm{Ba}$ & 1,63 \\
\hline 5. trifluralin & 2,10 & $1,50 \mathrm{Da}$ & $1,75 \mathrm{Ba}$ & 1,63 \\
\hline 6. oxyfluorfen & 0,24 & $2,25 \mathrm{Ca}$ & 3,00 Aa & 2,63 \\
\hline 7. alachlor + diuron & $1,68+1,75$ & $5,25 \mathrm{Aa}$ & $1,75 \mathrm{Bb}$ & 3,50 \\
\hline 8. alachlor + prometryne & $1,68+1,75$ & $5,50 \mathrm{Aa}$ & $2,25 \mathrm{Bb}$ & 3,88 \\
\hline 9. S-metolachlor + diuron & $0,96+1,75$ & 4,75 Aa & $2,00 \mathrm{Bb}$ & 3,38 \\
\hline 10. S-metolachlor + prometryne & $0,96+1,75$ & $5,00 \mathrm{Aa}$ & $2,00 \mathrm{Bb}$ & 3,50 \\
\hline 11. oxyfluorfen + diuron & $0,24+1,75$ & $2,25 \mathrm{Cb}$ & $3,50 \mathrm{Aa}$ & 2,88 \\
\hline 12. oxyfluorfen + prometryne & $0,24+1,75$ & $3,25 \mathrm{Ba}$ & $3,00 \mathrm{Aa}$ & 3,13 \\
\hline 13. trifluralin + diuron & $2,10+1,75$ & $1,25 \mathrm{Da}$ & $2,00 \mathrm{Ba}$ & 1,63 \\
\hline 14. trifluralin + prometryne & $2,10+1,75$ & $1,25 \mathrm{Da}$ & $2,00 \mathrm{Ba}$ & 1,63 \\
\hline 15. trifluralin + oxyfluorfen & $2,10+0,24$ & $2,00 \mathrm{Cb}$ & 3,00 Aa & 2,50 \\
\hline 16. testemunha sem herbicida & - & $1,00 \mathrm{Da}$ & $1,00 \mathrm{Ba}$ & 1,00 \\
\hline Médias & & 2,78 & 2,14 & \\
\hline C.V. $(\%)$ & 26,32 & & & \\
\hline
\end{tabular}

Médias seguidas de mesma letra maiúscula na coluna e minúscula na linha não diferem entre si pelo teste de agrupamento Scott-Knott (1974) a 5\%

A Tabela 7 mostra que, em ambos os locais, as plantas ainda apresentavam injúrias aos 29 DAA. Em Diamantino, as plantas provenientes dos tratamentos com alachlor, alachlor + prometryne, S-metolachlor + prometryne, oxyfluorfen + prometryne e trifluralin + prometryne ainda apresentavam sintomas de fitointoxicação. Em Campos de Júlio, os tratamentos oxyfluorfen, oxyfluorfen + diuron, oxyfluorfen + prometryne e trfluralin + oxyfluorfen proporcionaram os maiores valores de fitointoxicação, em relação à testemunha. Yamashita et al. (2008) também observaram danos ao algodoeiro até os 35 DAA, com a utilização de $180 \mathrm{~g} \mathrm{ha}^{-1}$ de oxyfluorfen, com substrato coletado em área de mata (29 $\mathrm{g} \mathrm{dm}^{-3}$ de matéria orgânica).

Para a variável fitointoxicação de plantas aos 36 DAA, a análise de variância conjunta dos dados revelou efeito não significativo $(\mathrm{p}>0,05)$ para a interação tratamentos x locais. A média de fitointoxicação dos herbicidas em Diamantino $(1,5)$ foi superior, em relação à de Campos de Júlio $(1,1)$. Tal fato pode estar relacionado aos menores teores de argila e de matéria orgânica, que o solo de Diamantino possui em relação a Campos de
Júlio (Tabela 1), o que favorece a menor retenção das moléculas dos herbicidas na camada superficial do solo (INOUE et al., 2009).

No estande de plantas avaliado aos 21 e 49 DAA, a análise de variância conjunta dos dados revelou efeito não significativo $(\mathrm{p}>0,05)$ para a interação tratamentos $\mathrm{x}$ locais e os tratamentos com herbicidas não afetaram o estande de plantas. No entanto, em ambas as datas de avaliação, Campos de Júlio apresentou estande médio de plantas mais elevado (21 DAA = 9,05 plantas; $49 \mathrm{DAA}=$ $9,02$ plantas $)$, em relação à Diamantino (21 DAA $=7,74$ plantas; 49 DAA = 6,93 plantas). Tal resultado pode ser atribuído à porcentagem de germinação das sementes, pois a semente utilizada em Diamantino apresentou $88 \%$ de germinação e a de Campos de Júlio 93\%.

Com relação ao número de maçãs, não houve diferença significativa entre os tratamentos ou entre locais. Apesar das injúrias visuais observadas inicialmente e do efeito de alguns tratamentos sobre o crescimento inicial das plantas de algodão, nenhum dos tratamentos com herbicidas foi suficientemente tóxico para afetar o número de maçãs por planta, em ambas as localidades. Do mesmo 
Tabela 7 - Avaliação de fitointoxicação aos 29 dias após aplicação (DAA) dos tratamentos com herbicidas em pré-emergência. Diamantino e Campos de Júlio - MT. Safra 2008/2009

\begin{tabular}{lcccc}
\hline \multirow{2}{*}{ Tratamentos (i.a.) } & \multirow{2}{*}{ kg i.a. ha $^{-1}$} & \multicolumn{2}{c}{ Fitointoxicação } & \multirow{2}{*}{ Médias } \\
\cline { 2 - 4 } & 1,68 & $2,25 \mathrm{Aa}$ & $1,50 \mathrm{Ba}$ & 1,88 \\
1. alachlor & 0,96 & $1,75 \mathrm{Ba}$ & $1,50 \mathrm{Ba}$ & 1,63 \\
2. S-metolachlor & 1,75 & $1,75 \mathrm{Ba}$ & $1,25 \mathrm{Ba}$ & 1,50 \\
3. diuron & 1,75 & $1,75 \mathrm{Ba}$ & $2,00 \mathrm{Ba}$ & 1,88 \\
4. prometryne & 2,10 & $1,75 \mathrm{Ba}$ & $1,75 \mathrm{Ba}$ & 1,75 \\
5. trifluralin & 0,24 & $1,75 \mathrm{Bb}$ & $3,00 \mathrm{Aa}$ & 2,38 \\
6. oxyfluorfen & $1,68+1,75$ & $2,00 \mathrm{Ba}$ & $1,75 \mathrm{Ba}$ & 1,88 \\
7. alachlor + diuron & $1,68+1,75$ & $2,50 \mathrm{Aa}$ & $1,75 \mathrm{Ba}$ & 2,13 \\
8. alachlor + prometryne & $0,96+1,75$ & $1,75 \mathrm{Ba}$ & $1,50 \mathrm{Ba}$ & 1,63 \\
9. S-metolachlor + diuron & $0,96+1,75$ & $2,50 \mathrm{Aa}$ & $1,50 \mathrm{Bb}$ & 2,00 \\
10. S-metolachlor + prometryne & $1,75 \mathrm{Bb}$ & $3,00 \mathrm{Aa}$ & 2,38 \\
11. oxyfluorfen + diuron & $0,24+1,75$ & $2,25 \mathrm{Aa}$ & $3,00 \mathrm{Aa}$ & 2,63 \\
12. oxyfluorfen + prometryne & $0,24+1,75$ & $1,75 \mathrm{Ba}$ & $1,75 \mathrm{Ba}$ & 1,75 \\
13. trifluralin + diuron & $2,10+1,75$ & $2,50 \mathrm{Aa}$ & $1,75 \mathrm{Ba}$ & 2,13 \\
14. trifluralin + prometryne & $2,10+1,75$ & $2,00 \mathrm{Bb}$ & $3,00 \mathrm{Aa}$ & 2,50 \\
15. trifluralin + oxyfluorfen & $2,10+0,24$ & $1,00 \mathrm{Ba}$ & $1,00 \mathrm{Ba}$ & 1,00 \\
16. testemunha sem herbicida & - & 1,94 & 1,94 & \\
\hline \multicolumn{1}{c}{ Médias } & \multicolumn{5}{c}{ C.V. (\%) } & 28,07 &
\end{tabular}

Médias seguidas de mesma letra maiúscula na coluna e minúscula na linha não diferem entre si pelo teste de agrupamento Scott-Knott (1974) a 5\%

modo, Arantes (2008) ao utilizar tratamentos herbicidas semelhantes aos utilizados neste trabalho aplicados em pré-emergência em Argissolo Vermelho distrófico de textura média, também não constatou diferenças no número de maçãs por planta determinado aos 141 DAA na cultivar de algodão FMT-701.

Os dados de produtividade indicam que todos os herbicidas foram seletivos ao algodoeiro, em relação à respectiva testemunha de cada localidade (Tabela 8). Quanto aos danos fitotóxicos, as plantas se recuperaram e apresentaram produtividade semelhante à testemunha. Isso se deve ao longo ciclo que a cultura possui, tendo assim tempo para se recuperar não apresentando diferenças na produtividade.

A ausência de efeitos significativos dos tratamentos com herbicidas, em relação às respectivas testemunhas de cada local, indica que os efeitos dos tratamentos em relação ao crescimento das plantas não são definitivos, ou seja, mesmo plantas que apresentaram menor crescimento nas primeiras avaliações apresentaram recuperação ao longo do ciclo. Vieira et al. (1999) ao testarem diuron e alachlor nas doses 2,25+1,44 $\mathrm{kg} \mathrm{ha}^{-1}$ em Vertissolo Textura Franco
- Limosa, não observaram influência dos herbicidas no rendimento da cultura sobre a cultivar de algodão CNPA 7H. Santana (2008) ao avaliar a seletividade de clomazone $\left(0,9 \mathrm{~kg} \mathrm{ha}^{-1}\right)$, S-metolachlor $\left(0,672 \mathrm{~kg} \mathrm{ha}^{-1}\right)$, diuron $(0,9$ $\left.\mathrm{kg} \mathrm{ha}^{-1}\right)$, prometryne $\left(0,9 \mathrm{~kg} \mathrm{ha}^{-1}\right)$, alachlor $\left(1,2 \mathrm{~kg} \mathrm{ha}^{-1}\right)$ e oxyfluorfen $\left(0,192 \mathrm{~kg} \mathrm{ha}^{-1}\right)$, em Argissolo Vermelho Distrófico, não constatou diferença de produtividade entre os tratamentos e suas respectivas testemunhas adjacentes sobre a cultivar de algodão FMT-701. Em contra partida, Dan et al. (2011) relatam que houve queda significativa na produtividade para as misturas em tanque de clomazone + oxyfluorfen $\left(1,00+0,19 \mathrm{~kg} \mathrm{ha}^{-1}\right)$, clomazone + trifluralin + diuron $\left(1,25+1,80+1,50 \mathrm{~kg} \mathrm{ha}^{-1}\right)$ e clomazone + trifluralin + prometryne $\left(1,25+1,80+1,50 \mathrm{~kg} \mathrm{ha}^{-1}\right)$ para a cultivar Nu- Opal em Latossolo Vermelho distroférrico.

Ao comparar a produtividade de ambos os locais, notou-se que em Campos de Júlio a produtividade foi mais elevada (Tabela 8). Tal fator está ligado ao estande que foi mais elevado em Campos de Júlio em relação a Diamantino, além das possíveis características edafoclimáticas da região e as características do solo, o qual possuía maior porcentagem de argila em relação à Diamantino (Tabela 1). 
Tabela 8 - Avaliação da produtividade $\left(\mathrm{kg} \mathrm{ha}^{-1}\right)$ aos 193 dias após aplicação (DAA) dos tratamentos com herbicidas em pré-emergência. Diamantino e Campos de Júlio - MT. Safra 2008/2009

\begin{tabular}{lcccc}
\hline \multicolumn{1}{c}{ Tratamentos (i.a.) } & kg i.a. ha-1 & \multicolumn{2}{c}{ Produtividade (kg ha-1) } & Médias \\
\cline { 3 - 4 } 1. alachlor & 1,68 & Diamantino & C. de Júlio & 3455,25 \\
2. S-metolachlor & 0,96 & $3410,49 \mathrm{Aa}$ & $3500,00 \mathrm{Aa}$ & 3435,19 \\
3. diuron & 1,75 & $3333,33 \mathrm{Aa}$ & $3537,04 \mathrm{Aa}$ & 3498,46 \\
4. prometryne & 1,75 & $3219,14 \mathrm{Ab}$ & $3777,78 \mathrm{Aa}$ & 3433,64 \\
5. trifluralin & 2,10 & $3256,17 \mathrm{Aa}$ & $3611,11 \mathrm{Aa}$ & 3375,00 \\
6. oxyfluorfen & 0,24 & $3231,48 \mathrm{Aa}$ & $3518,52 \mathrm{Aa}$ & 3212,97 \\
7. alachlor + diuron & $1,68+1,75$ & $3148,15 \mathrm{Aa}$ & $3277,78 \mathrm{Aa}$ & 3576,54 \\
8. alachlor + prometryne & $1,68+1,75$ & $3271,60 \mathrm{Ab}$ & $3881,48 \mathrm{Aa}$ & 3461,42 \\
9. S-metolachlor + diuron & $0,96+1,75$ & $3256,17 \mathrm{Aa}$ & $3666,67 \mathrm{Aa}$ & 3455,25 \\
10. S-metolachlor + prometryne & $0,96+1,75$ & $3410,49 \mathrm{Aa}$ & $3500,00 \mathrm{Aa}$ & 3243,83 \\
11. oxyfluorfen + diuron & $0,24+1,75$ & $3024,69 \mathrm{Ab}$ & $3462,96 \mathrm{Aa}$ & 3260,81 \\
12. oxyfluorfen + prometryne & $0,24+1,75$ & $3354,94 \mathrm{Aa}$ & $3166,67 \mathrm{Aa}$ & 3310,80 \\
13. trifluralin + diuron & $2,10+1,75$ & $3349,12 \mathrm{Aa}$ & $3322,22 \mathrm{Aa}$ & 3489,38 \\
14. trifluralin + prometryne & $2,10+1,75$ & $3414,26 \mathrm{Aa}$ & $3333,33 \mathrm{Aa}$ & 3373,80 \\
15. trifluralin + oxyfluorfen & $2,10+0,24$ & $3397,12 \mathrm{Aa}$ & $3370,37 \mathrm{Aa}$ & 3383,75 \\
16. testemunha sem herbicida & - & $3365,43 \mathrm{Aa}$ & $3494,32 \mathrm{Aa}$ & 3429,88 \\
\hline \multicolumn{1}{c}{ Médias } & 8,47 & 3296,37 & 3503,12 & \\
\hline
\end{tabular}

Médias seguidas de mesma letra maiúscula na coluna e minúscula na linha não diferem entre si pelo teste de agrupamento Scott-Knott (1974), a 5\%

\section{CONCLUSÃO}

Todos os tratamentos foram seletivos à cultura do algodão cultivar FMT-701 nas duas localidades, sendo que mesmo àqueles que proporcionaram fitointoxicação inicial não causaram perdas na produtividade.

\section{REFERÊNCIAS}

ARANTES, J. G. Z. Seletividade de herbicidas aplicados em pré-emergência na cultura do algodoeiro (Gossypium hirsutum L.). 2008. 64 f. Dissertação (Mestrado em Agronomia) - Universidade Estadual de Maringá, Maringá, 2008.

AZEVEDO, D. M. P. et al. Estudo da eficiência da mistura de alachlor/diuron no controle de plantas daninhas em algodoeiro anual irrigado. Pesquisa Agropecuária Brasileira, v. 28, n. 7, p. $779-785,1993$.

BALLAMINUT, C. E. C. Seletividade da cultura do algodoeiro aos herbicidas diuron, clomazone, trifloxysulfuron-sodium e pyrithiobac-sodium. 2009. 86 f. Dissertação (Mestrado em Agronomia) - Escola Superior de Agricultura "Luiz de Queiroz", Piracicaba, 2009.
BANZATTO, D. A.; KRONKA, S. N. Experimentação Agrícola. 3. ed. Jaboticabal: FUNEP, 1995. 247 p.

CARDOSO, G. D. et al. Períodos de interferencia das plantas dañinas em algodoeiro de fibra colorida 'BRS Safira'. Revista Ciência Agronômica, v. 41, n. 3, p. 456, 2010.

COMPANHIA NACIONAL DE ABASTECIMENTO. Acompanhamento da safra brasileira: Grãos: safra 2010/2011: Décimo segundo levantamento. 2011. 41 p. Disponível em: <http://www.conab.gov.br/OlalaCMS/uploads/ arquivos/11_09_19_09_49_47_boletim_setembro-2011..pdf $>$. Acesso em: 1 fev. 2011.

CRUSCIOL, C. A. C. et al. Aplicação tardia de glyphosate e estande e desenvolvimento inicial do arroz em sistemas de cultivo mínimo. Planta Daninha, v. 20, n. 1, p. 45-51, 2002.

EMPRESA BRASILEIRA DE PESQUISA AGROPECUÁRIA. Sistema brasileiro de classificação de solos. 2. ed. Rio de Janeiro: EMBRAPA, 2006. 306 p.

DAN, H. A. et al. Seletividade de clomazone isolado ou em mistura para a cultura do algodoeiro. Planta Daninha, v. 29, n. 3, p. 601-607, 2011.

FERREIRA, J. C. V; SILVA, J. M. Cidades de Mato Grosso. 2. ed. Cuiabá: Memória Brasileira, 2008. 240 p. 
FOLONI, L. L.; RODRIGUES, J. D.; ONO, E. O. Avaliação de tratamentos químicos e mecânicos no controle de plantas daninhas na cultura do algodão. Planta Daninha, v. 17, n. 1, p. 5-20, 1999.

FREITAS, R. S. et al. Manejo de plantas daninhas na cultura do algodoeiro com S-metolachlor e trifloxysulfuron-sodium em sistema de plantio convencional. Planta Daninha, v. 24, n. 2, p. 311-318, 2006a.

FREITAS, R. S. et al. Manejo de plantas daninhas na cultura do algodoeiro em sistema de plantio direto. Planta Daninha, v. 24, n. 2, p. 339-346, 2006 b.

FREITAS, R. S. Interferência de plantas daninhas na cultura do algodão. Revista Ceres, v. 44, n. 256, p. 597-603, 2003.

GOULART, A. C. P. Efeito do tratamento de sementes de algodão com fungicidas no controle do tombamento de plântulas causado por Rhizoctonia solani. Fitopatologia Brasileira, v. 27, n. 4, p. 399-402, 2002.

GUIMARÃES, S. C.; HRYCYK, M. F.; MENDONÇA, E. A. F. Efeito de fatores ambientais sobre a seletividade do alachlor ao algodoeiro. Planta Daninha, v. 25, n. 4, p. 813821, 2007.

INOUE, M. H. et al. Bioavailability of diuron, imazapic and isoxaflutole in soils of contrasting textures. Journal of Environmental Science and Health, Part B, v. 44, n. 8, p. 757$763,2009$.
INOUE, M. H. et al. Efeito residual de herbicidas aplicados em pré-emergência em diferentes solos. Planta Daninha, v. 29, n. 2, p. 429-435, 2011.

INOUE, M. H. et al. Potencial de lixiviação de herbicidas utilizados na cultura do algodão em colunas de solo. Planta Daninha, v. 28, n. 4, p. 825-833, 2010.

SANTANA, S. C. B. Seletividade de clomazone, isolado ou em mistura com outros herbicidas, para dois cultivares de algodoeiro. 2008. 57 f. Dissertação (Mestrado em Agronomia) - Universidade Estadual de Maringá, Maringá, 2008.

SCOTT, A. J.; KNOTT, M. A. A cluster analysis method for grouping means in the analysis of variance. Biometrics, v. 30, p. 507-512, 1974.

SILVA, A. F. et al. Interceptação da luz, matéria seca e área foliar de linhagens de algodoeiro herbáceo. Revista Ciência Agronômica, v. 36, n. 1, p. 67-73, 2005.

SOCIEDADE BRASILEIRA DA CIÊNCIA DAS PLANTAS DANINHAS. Procedimentos para instalação, avaliação e análise de experimentos com herbicidas. Londrina: SBCPD, 1995. 42 p.

VIEIRA, D. J. et al. Misturas de herbicidas e populações de plantas no controle de plantas daninhas em algodoeiro herbáceo. Revista Oleaginosas e Fibrosas, v. 3, n. 1, p. 143-152, 1999.

YAMASHITA, O. M. et al. Efeito de doses reduzidas de oxyfluorfen em cultivares de algodoeiro. Planta Daninha, v. 26, n. 4, p. 917-921, 2008. 